\title{
Ultrafast high-fidelity initialization of a quantum-dot spin qubit without magnetic fields
}

\author{
Jonathan D. Mar, ${ }^{1,2,{ }^{*}}$ Jeremy J. Baumberg, ${ }^{2}$ Xiulai Xu, ${ }^{1, \dagger}$ Andrew C. Irvine, ${ }^{3}$ and David A. Williams ${ }^{1}$ \\ ${ }^{1}$ Hitachi Cambridge Laboratory, Cavendish Laboratory, Cambridge CB3 OHE, United Kingdom \\ ${ }^{2}$ NanoPhotonics Centre, Cavendish Laboratory, University of Cambridge, Cambridge CB3 OHE, United Kingdom \\ ${ }^{3}$ Microelectronics Research Centre, Cavendish Laboratory, University of Cambridge, Cambridge CB3 OHE, United Kingdom
}

(Received 13 June 2014; published 15 December 2014)

\begin{abstract}
We demonstrate the initialization of a single quantum-dot hole spin with high fidelity (lower bound $>97 \%$ ), on picosecond time scales, and without the need for magnetic fields. Using the initialization scheme based on rapid electric-field ionization of a resonantly excited exciton, this is achieved by employing a self-assembled quantum dot with a low conduction-to-valence band offset ratio, allowing control of the relative electron and hole tunneling rates over three orders of magnitude. This large difference in tunneling rates could permit spin-storage efficiencies $>99.5 \%$ by fast-switching to a low electric-field condition. Our results may provide a practical route towards ultrafast high-fidelity initialization of individual quantum-dot hole spins for the implementation of quantum error correction in a scalable spin-based quantum computer.
\end{abstract}

DOI: 10.1103/PhysRevB.90.241303

PACS number(s): 73.21.La, 73.63.-b, 78.67.-n

The spin of an electron or hole confined in a semiconductor quantum dot $(\mathrm{QD})$ is a promising candidate for a qubit-the building block of a quantum computer-due to its long relaxation and decoherence times [1-3] and scalability into large arrays. An essential requirement for quantum computing based on such qubits is the ability to initialize individual spins with high fidelity and within a scalable quantum device [4]. A further requirement is that, in order to perform quantum error correction necessary for fault-tolerant quantum computing, certain qubits must be continuously reinitialized on ultrafast time scales [4,5], since the decoherence time-beyond which information stored in a qubit is lost-is approximately $100 \mathrm{~ns}$ [6] (1 $\mu \mathrm{s}$ [7]) for the free-induction (spin-echo) decay of a hole spin in a self-assembled QD and approximately $1 \mu \mathrm{s}[8,9](200 \mu \mathrm{s}$ [10]) for the spin-echo decay of an electron spin in a self-assembled (electrostatically defined) QD. Highfidelity initialization of QD spins has been demonstrated previously using various methods [10-15]. However, initialization rates were too slow [10-15] for implementing error correction and/or achieving high fidelities necessitated applied magnetic fields $[10,12-15]$. The need to apply magnetic fields greatly compromises the scalability of a quantum device into large quantum circuits due to additional cost and space requirements $[16,17]$. Further, with the recent demonstration of optical coherent manipulation and readout of a QD spin without applied magnetic fields [18], initialization without magnetic fields has gained increased interest. Additionally, it has been shown that hole-spin relaxation in a QD due to interactions with phonons is eliminated in the limit of zero magnetic field $[11,19,20]$. Here we demonstrate the ultrafast initialization of a single-QD hole spin on picosecond time scales, with high fidelity (lower bound $>97 \%$ ), and without the need for magnetic fields. Using an initialization scheme based on rapid electric-field ionization of a resonantly

\footnotetext{
*Author to whom correspondence should be addressed: jm585@cam.ac.uk

${ }^{\dagger}$ Present address: Beijing National Laboratory for Condensed Matter Physics, Institute of Physics, Chinese Academy of Sciences, Beijing 100190, People's Republic of China.
}

excited exciton, this is achieved by employing a QD with a low conduction-to-valence band offset ratio, allowing control of the relative electron and hole tunneling rates over three orders of magnitude. A theoretical model based on measured parameters provides evidence for an achieved fidelity of near unity (>99.9999\%). Further, the large difference in tunneling rates could permit spin-storage efficiencies $>99.5 \%$ by fast-switching to a low electric-field condition. Compared to electron spins, hole spins should be more favorable as qubits since their hyperfine interaction with the nuclear spin ensemble leading to decoherence is greatly suppressed [19].

The scheme used here for initialization of a single hole spin [21] is illustrated in Fig. 1. With the QD initially empty of carriers, a $\sigma^{+/-}$circularly polarized laser field resonantly excites a neutral exciton $\left(X^{0}\right)$ with spin $M_{z}= \pm 1$, consisting of a spin-up (-down) heavy hole with spin $J_{z}= \pm 3 / 2$ and a spin-down (-up) electron with spin $S_{z}=\mp 1 / 2$. In the presence of an electric field, this is followed by rapid ionization of $X^{0}$ via electron tunneling at a rate $\Gamma_{e}$, since the effective mass of an electron is much smaller than that of a heavy hole, thereby leaving a single hole in the QD initialized with a known spin, which was determined by the laser polarization. For example, $\sigma^{+}$-polarized excitation followed by exciton ionization results in the initialization of a spin-up hole. Prior to ionization via electron tunneling, however, $X^{0}$ spin precession between states $M_{z}= \pm 1$ due to exciton fine-structure splitting $\left(\delta_{\mathrm{FS}}\right)$, which is caused by an anisotropic electron-hole $(e-h)$ exchange energy in self-assembled QDs resulting in linearly polarized eigenstates [22], leads to a loss in hole-spin initialization fidelity. Therefore, an essential requirement for achieving high-fidelity initialization under this scheme is that $\Gamma_{e}$, which also determines the spin initialization rate, be much greater than the rate of spin dephasing, $\delta_{\mathrm{FS}} / h$. Then, to measure the initialization fidelity for the desired hole-spin state, a second circularly polarized laser field resonant with the positive trion state $\left(X^{+}\right)$, which is composed of two holes in a spin singlet and an electron with either spin, is used to compare the probabilities of exciting $X^{+}$for cross- and copolarized excitation relative to the first laser: Excitation of $X^{+}$from a given hole-spin state is conditional on the polarization of the second laser due to optical selection rules (Fig. 1). For example, for initialization 


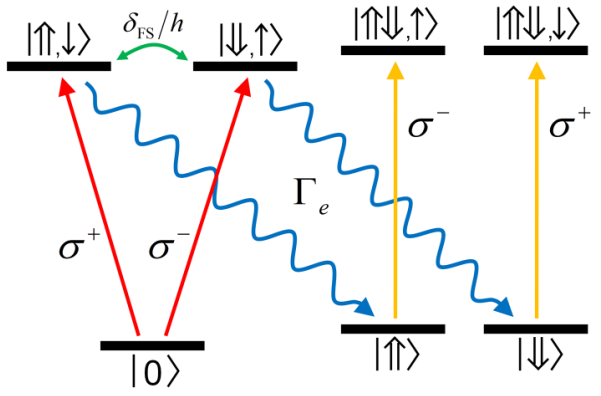

FIG. 1. (Color online) Scheme for hole-spin initialization and measurement of fidelity. Resonant excitation from the ground state $|0\rangle$ (empty QD) to the $X^{0}$ state $|\Uparrow, \downarrow\rangle(|\Downarrow, \uparrow\rangle)$, which consists of a spin-up (-down) hole and a spin-down (-up) electron, is achieved using a $\sigma^{+}\left(\sigma^{-}\right)$-polarized laser (straight arrows on left-hand side). Fast ionization of $|\Uparrow, \downarrow\rangle(|\Downarrow, \uparrow\rangle)$ via electron tunneling results in the initialization of $|\Uparrow\rangle(|\Downarrow\rangle)$ at a rate $\Gamma_{e}$ (wavy arrows). Prior to electron tunneling, however, coupling between $X^{0}$ states due to fine-structure splitting at a frequency $\delta_{\mathrm{FS}} / h$ leads to $X^{0}$ spin dephasing and a subsequent loss in initialization fidelity. Using a second laser tuned to the $X^{+}$transition, the fidelity of $|\Uparrow\rangle(|\Downarrow\rangle)$ initialization is measured by comparing the probability of creating $X^{+}$for $\sigma^{-}\left(\sigma^{+}\right)$cross-polarized and $\sigma^{+}\left(\sigma^{-}\right)$copolarized excitation with respect to the polarization of the first laser.

of a spin-up hole using a $\sigma^{+}$-polarized laser tuned to the $X^{0}$ transition, the initialization fidelity is measured by comparing the probabilities of exciting $X^{+}$for a $\sigma^{-}$- and $\sigma^{+}$-polarized laser tuned to the $X^{+}$transition, where the former (latter) represents occupation of the spin-up (-down) hole state.

To implement the above initialization scheme, our device consists of a single layer of InAs/GaAs self-assembled QDs grown by molecular beam epitaxy, embedded in the intrinsic region of an $n-i$-Schottky photodiode structure, and characterized by a low surface density of QDs $\left(\sim 10^{9} \mathrm{~cm}^{-2}\right)$ suitable for performing single-QD optical measurements. Such a device structure enables electric-field-tunable carrier tunneling out of the QD or bias-controlled single-electron charging when the QD $s$ shell (lowest-energy) electron level is shifted above or below the Fermi level $E_{\mathrm{F}}$, respectively, via an appropriate tuning of the bias voltage $V_{\mathrm{b}}$ [23-26]. Initial measurements on single QDs are performed using biasdependent micro-photoluminescence ( $\mu$-PL) spectroscopy, via above-band gap laser excitation, in order to identify straightforwardly the transition energies of $X^{0}, X^{+}$, the singly negatively charged exciton state, and the biexciton state $[23,24]$. Such identification of the relevant transition energies allows for high-resolution measurements of the $X^{0}$ $\left(X^{+}\right)$absorption spectrum via photocurrent (PC) spectroscopy using one (two) resonant narrow-bandwidth independently tunable continuous-wave laser(s) [24,25] ([26]). It should be noted that all measurements in this work were performed at a sample temperature of $4.2 \mathrm{~K}$. Further details of the device structure and fabrication, bias-dependent $\mu$-PL spectroscopy, the experimental setup for high-resolution single-QD PC spectroscopy, and experimental methods and techniques used for PC measurements of the $X^{0}$ and $X^{+}$absorption spectra, can be found in the Supplemental Material [27].
As pointed out earlier, the initialization scheme used here requires that $\delta_{\mathrm{FS}} / h \ll \Gamma_{e}$ in order to achieve fast high-fidelity initialization without the need for an applied magnetic field. As important, the ability to perform efficient spin storage via fastswitching to a low reverse-bias voltage at a time sufficiently later (earlier) than electron (hole) tunneling requires that the hole tunneling rate $\Gamma_{h} \ll \Gamma_{e}$. To satisfy these conditions, we use QDs having a low conduction-to-valence band offset ratio, which can be found in QDs with reduced heights and thus characterized by relatively high transition energies. This can be understood by considering the QD as a one-dimensional (1D) potential well (in the direction of the applied electric field and carrier tunneling) with quantized energy levels $E_{n}=$ $(n \hbar \pi)^{2} / 2 m_{e / h}^{*} H^{2}$, where $m_{e / h}^{*}$ is the electron/heavy-hole effective mass, $H$ is the QD height (i.e., potential-well width), and $n$ is the energy-level index. Thus, since $m_{e}^{*}=0.067 m_{e}$ [28] is nearly an order of magnitude smaller than $m_{h}^{*}=0.59 m_{e}$ [29] in GaAs, where $m_{e}$ is the electron mass in vacuum, reducing $H$ results in a decrease in the ratio of conduction- to valence-band offsets. This in turn leads to an increase in the ratio of electron to hole tunneling rates, which vary exponentially with their respective band offsets. Since reducing $H$ also results in an increase in the QD transition energies, we therefore expect to satisfy the above conditions in the case of high-energy QDs. Additionally, it has been observed that such small, high-energy QDs intrinsically possess small values of $\delta_{\mathrm{FS}}[30,31]$. Such an effect is due to an increased in-plane expansion of the exciton, as a result of the decreased confinement energy (i.e., band offset), leading to a reduction in the $e-h$ exchange energy and, hence, $\delta_{\mathrm{FS}}[32]$.

We search for small, high-energy QDs near the critical InAs thickness for QD formation [30,31], which is also where a low surface density of QDs is obtained to allow for optical measurements on single QDs. We select a suitable single QD with an $X^{0}$ transition energy of $\sim 1.364 \mathrm{eV}$. As a function of $V_{\mathrm{b}}$ (i.e., vertical electric field $F$ ), we then measure $\Gamma_{e}$ from the laser-intensity-dependent $X^{0}$ homogeneous linewidth using PC spectroscopy [24], $\Gamma_{h}$ from the saturation of the $X^{0}$ PC peak amplitude towards high laser intensities [25], and $\delta_{\mathrm{FS}}$ from PC spectroscopy of each of the $X^{0}$ fine-structure split eigenstates using a linearly polarized laser aligned either to the [110] or [1-10] crystallographic axis [22] (see Supplemental Material [27]). The results shown in Fig. 2 reveal that indeed $\Gamma_{e}$ is about three orders of magnitude faster than $\Gamma_{h}$ throughout the measurable PC range for $X^{0}$. Also, fitting a theoretical model based on a 1D Wentzel-Kramers-Brillouin approximation $[24,25,33]$ to the experimental data for $\Gamma_{e}$ and $\Gamma_{h}$ (Fig. 2, solid lines), we extract a conduction-to-valence band offset ratio of $1.29(=52.2 / 40.5 \mathrm{meV} / \mathrm{meV})$ for this particular QD [24,25]. These results are in contrast to those reported in other works also using $\mathrm{In}(\mathrm{Ga}) \mathrm{As} / \mathrm{GaAs}$ self-assembled QDs but with much lower transition energies, where the ratio of $\Gamma_{e}$ to $\Gamma_{h}$ is nearly a factor of $10^{2}$ smaller [15,21,34] and the conduction-to-valence band offset ratio is nearly a factor of 2 larger [35,36]. Figure 2 also shows that $\Gamma_{e}$ is between two and four orders of magnitude larger than $\delta_{\mathrm{FS}} / h$ over the measurable $\mathrm{PC}$ range. The observed decrease in $\delta_{\mathrm{FS}}$ with increasing $F$, which corresponds to a reduction of $\delta_{\mathrm{FS}}$ from $\sim 18 \mu \mathrm{eV}$ to $\sim 1 \mu \mathrm{eV}$, is most likely the result of an in-plane symmetrizing and/or vertical spatial separation of electron and 


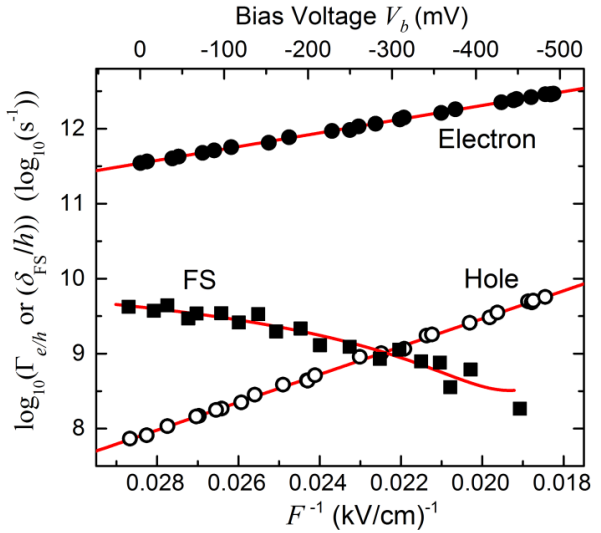

FIG. 2. (Color online) Comparison of electron tunneling rate to hole tunneling rate and fine-structure splitting. $\Gamma_{e}$ is about three orders of magnitude faster than $\Gamma_{h}$ throughout the measured range. Also, $\Gamma_{e}$ is between two and four orders of magnitude faster than $\delta_{\mathrm{FS}} / h$ throughout the measured range. For $\delta_{\mathrm{FS}}$, the solid curve is the difference between the quadratic fit curves of the $V_{\mathrm{b}}$ vs. energy data for the two linearly polarized $X^{0}$ eigenstates (see Supplemental Material [27]).

hole wave functions in the QD [37]. Finally, it is important to note that we are unable to perform these measurements on larger, lower-energy QDs for comparison, since such QDs can only be found towards areas on the wafer of increasing InAs thickness where the QD surface density becomes too large to allow for optical measurements on single QDs.

We are now in a position to measure the initialization fidelity that can be achieved using the scheme shown in Fig. 1. As discussed earlier, the fidelity is measured by comparing the $X^{+}$ PC peak amplitudes between cross- and cocircularly polarized excitation for the laser resonant to $X^{+}$relative to the laser resonant to $X^{0}$. For the initialization of a spin-up hole, the initialization fidelity is defined as $\mathrm{PC}_{+/-} /\left(\mathrm{PC}_{+/-}+\mathrm{PC}_{+/+}\right)$, where $\mathrm{PC}_{+/-}\left(\mathrm{PC}_{+/+}\right)$is the $X^{+} \mathrm{PC}$ peak amplitude for a $\sigma^{+}$-polarized $X^{0}$ laser and a $\sigma^{-}\left(\sigma^{+}\right)$-polarized $X^{+}$laser. An analogous definition for the initialization fidelity of a spin-down hole can be derived. As seen in Figs. 3(a) and 3(b), results show very high contrast in the $X^{+}$PC peak amplitudes between cross- and copolarized excitation in the case of both spin-up [Fig. 3(a)] and spin-down [Fig. 3(b)] initialization, which is expected since the two spin states are energetically degenerate in the absence of an applied magnetic field. The results here demonstrate lower bounds for the spin-up and spindown initialization fidelities of $97.1 \%$ and $96.9 \%$, respectively, at $V_{\mathrm{b}}=-104.4 \mathrm{mV}\left[F^{-1}=0.0249(\mathrm{kV} / \mathrm{cm})^{-1}\right]$. Notice that the PC peak has vanished below the noise level when the lasers are copolarized. Therefore, we report the fidelity in terms of a lower bound that is determined by the error in measured fidelity and in turn the experimental signal-to-noise ratio (SNR), given that a specific higher value for the achieved fidelity cannot be confirmed experimentally. Despite the possibility that there is a slight loss of laser polarization and/or of alignment (orthogonality) of the $X^{+}$laser's polarization axis relative to that of the $X^{0}$ laser for co- (cross-) polarized excitation, the resulting change in $X^{+}$PC signal still remains below the noise level and, thus, does not affect the reported lower
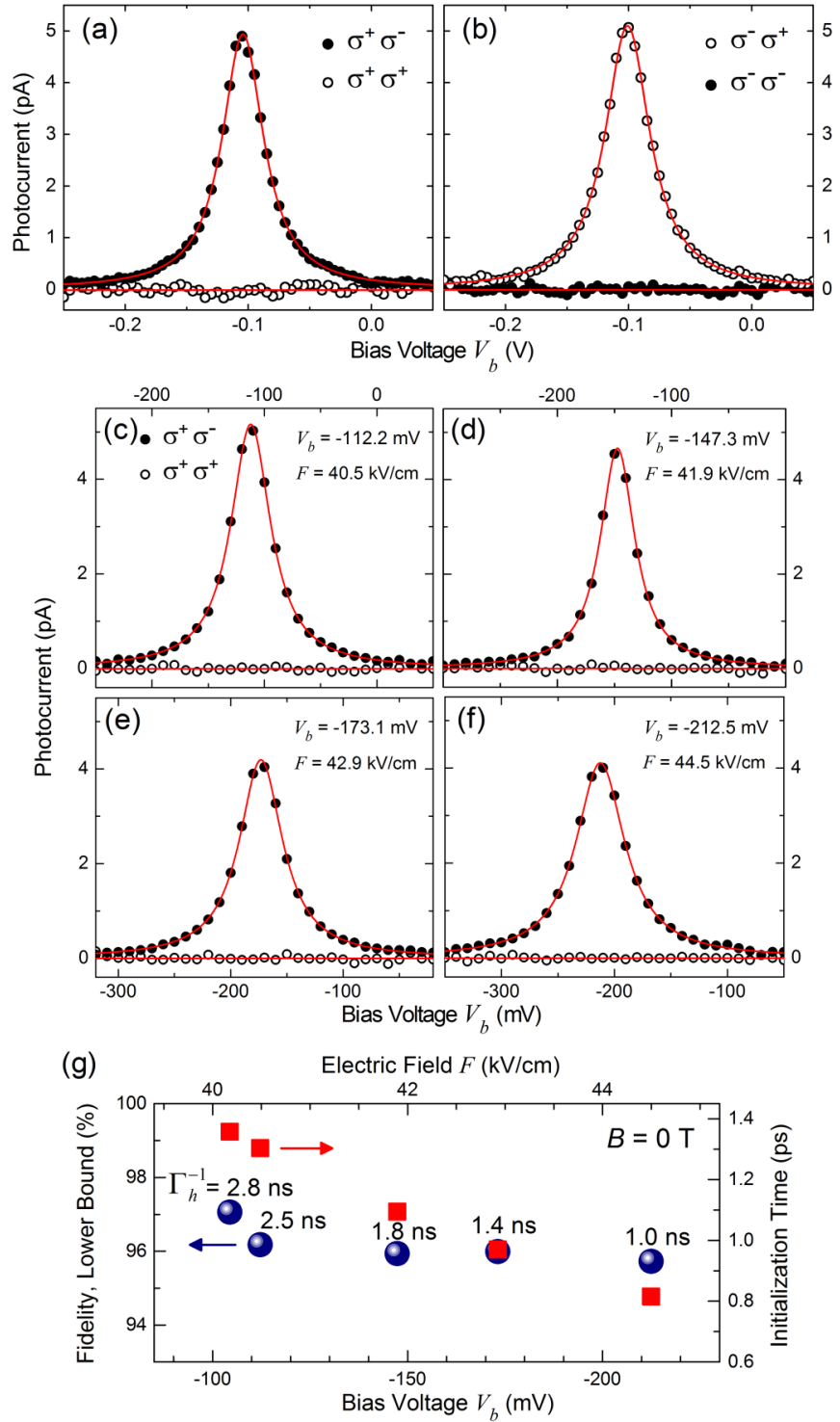

FIG. 3. (Color online) Measurement of the fidelity of hole-spin initialization. (a) $X^{+}$PC spectra for cross- (co-) polarized laser excitation using a $\sigma^{+}$-polarized $X^{0}$ laser, for the initialization of a spin-up hole, and a $\sigma^{-}\left(\sigma^{+}\right)$-polarized $X^{+}$laser. High contrast is observed as the copolarized PC peak vanishes below the noise level, thereby setting a lower bound for the fidelity. (b) Analogous measurement result to (a), but for the initialization of a spin-down hole. (c)-(f) Cross- and copolarized $X^{+} \mathrm{PC}$ spectra for a number of increasing reverse-bias voltages and for the initialization of a spin-up hole, showing similar results to (a) and (b). (g) Plot of the measured lower bounds of fidelity, along with their corresponding initialization time $\left(\Gamma_{e}^{-1}\right)$ and hole tunneling time $\Gamma_{h}^{-1}$, as a function of $V_{\mathrm{b}}$ and $F$. At $V_{\mathrm{b}}=-212.5 \mathrm{mV}$, the initialization time is reduced below $1 \mathrm{ps}$, while the lower bound of fidelity remains high, as seen also from the high contrast in (f), and $\Gamma_{h}^{-1}$ is still more than three orders of magnitude longer than $\Gamma_{e}^{-1}$ to allow for a potential spin-storage efficiency of near unity.

bounds of the fidelities. It is essential to point out that, for the peak $V_{\mathrm{b}}$ measured in Figs. 3(a) and 3(b), $\Gamma_{e}^{-1}=1.4 \mathrm{ps}$ and $\Gamma_{h}^{-1}=2.8 \mathrm{~ns}$ (Fig. 2). This means that, in addition to high-fidelity initialization, we have also demonstrated here an 
initialization time of $1.4 \mathrm{ps}$ and the potential for high-efficiency spin storage, as the hole has remained in the QD longer than the electron by more than three orders of magnitude. For example, if fast-switching to an appropriate low reverse-bias voltage is performed $10 \mathrm{ps}$ following the generation of $X^{0}$, a spin-storage efficiency of $99.6 \%$ can be achieved. In comparison, using the same voltage switching but now with a $\Gamma_{h}^{-1}$ only one order of magnitude longer than the above $\Gamma_{e}^{-1}$ (i.e., a ratio of $\Gamma_{e}$ to $\Gamma_{h}$ that is more than a factor of $10^{2}$ smaller), the spin-storage efficiency drops to $47.8 \%$.

As seen in Figs. 3(c)-3(f), similar high values for the lower bound of fidelity were also measured at a number of increased reverse-bias voltages for initialization of a spin-up hole. This is expected since, for an increasing reverse bias, the ratio of $\Gamma_{e}$ to $\delta_{\mathrm{FS}} / h$ increases (Fig. 2). The results are summarized in Fig. 3(g), along with their corresponding initialization time (i.e., $\Gamma_{e}^{-1}$ ) and $\Gamma_{h}^{-1}$. At $V_{\mathrm{b}}=-212.5 \mathrm{mV}$ $\left[F^{-1}=0.0225(\mathrm{kV} / \mathrm{cm})^{-1}\right]$, we observe the initialization time decrease well below 1 ps, while the lower bound of fidelity remains high $(95.7 \%)$ and $\Gamma_{h}^{-1}$ is still more than three orders of magnitude longer than $\Gamma_{e}^{-1}$ to allow for a high spin-storage efficiency of $99.0 \%$ when using the same voltage-switching operation as described above. We stress that, in all measurements, we have achieved high initialization fidelities without any magnetic fields $(B=0 \mathrm{~T})$. The slight decrease in the measured fidelity for increasing reverse-bias is simply the result of a decrease in the $X^{+}$PC peak amplitude for cross-polarized excitation due to an increase in $\Gamma_{h}$ and hence a decrease in the $X^{+}$photon absorption rate, rather than the result of changes in the initialization mechanisms involved. Indeed, according to the results in Fig. 2, the precise value of the fidelity should actually increase for increasing reverse-bias voltage, although this cannot be confirmed here experimentally. It was not possible to resolve this by simply increasing the $X^{+}$laser intensity used throughout our measurements, since this leads to a large increase in the noise level, greatly reducing the experimental SNR and, thus, the resulting lower bound of fidelity. It should also be pointed out that the fidelity was not measured for increasing reverse biases beyond those shown in Fig. 3, since the SNR and, hence, lower bound of fidelity decrease due to both an increasing background PC noise and a decreasing $X^{+}$photon absorption rate, while the potential spin-storage efficiency also begins to decrease (Fig. 2). On the other hand, the fidelity was not measured for decreasing reverse biases beyond those shown, since a decreasing $\Gamma_{h}$ leads to a vanishing PC peak amplitude, while the initialization time departs increasingly from the desired picosecond time scale. Finally, we note that we have also observed similar results for $\Gamma_{e}, \Gamma_{h}, \delta_{\mathrm{FS}}$, and an ensuing picosecond-time-scale high-fidelity hole-spin initialization, in several other high-energy single QDs in our sample.
Using rate equations for the spin-up and spin-down hole populations ( $h_{\Uparrow}$ and $h_{\Downarrow}$, respectively), a theoretical model can be derived for the contrast in $X^{+}$PC peak amplitude between co- and cross-circularly polarized excitation and, hence, for the fidelity of hole-spin initialization, in terms of $\delta_{\mathrm{FS}}, \Gamma_{e}$, $\Gamma_{h}$, and the Zeeman frequency $\omega_{z}$ for an applied magnetic field in the $z$ direction (i.e., Faraday configuration) [15]. For spin-up hole initialization, the contrast $=2 \times$ Fidelity $-1=$ $\lim _{\Gamma_{e} t \gg 1}\left(\frac{h_{\Uparrow}-h_{\Downarrow}}{h_{\Uparrow}+h_{\Downarrow}}\right)=1-\left[\frac{\left(\delta_{\mathrm{FS}} / h\right)^{2}}{\left(\delta_{\mathrm{FS}} / h\right)^{2}+\omega_{z}^{2}+\left(\Gamma_{e}-\Gamma_{h}\right)^{2}}\right]$, where $\omega_{z}$ is then set to zero, since we have not applied a magnetic field here. From our measured values for $\delta_{\mathrm{FS}}, \Gamma_{e}$, and $\Gamma_{h}$ (Fig. 2), the model predicts near-unity fidelity ( $>99.999 \%$ or, equivalently, a loss in fidelity of $<10^{-5}$ ) throughout the $V_{\mathrm{b}}$ range of our measured fidelities in Fig. 3(g). To the extent that the predicted values are within our measured lower bounds of fidelity, the above model agrees with our experimental data. Although the experimental SNR prevents us from confirming the precise theoretical fidelities, it is nevertheless encouraging to note that the model predicts that a fidelity of $>99.9999 \%$ or, equivalently, a loss in fidelity of $<10^{-6}$, can be achieved at $V_{\mathrm{b}}=-212.5 \mathrm{mV}$, which corresponds to the data point towards high reverse biases in Fig. 3(g).

We have successfully demonstrated picosecond-time-scale initialization of a QD hole-spin qubit with near-unity fidelity, in the absence of applied magnetic fields, and with the potential for near-unity spin-storage efficiency. Therefore, knowing from the results in Fig. 2 that $\Gamma_{h}$ can be tuned also below a picosecond using realistic bias voltages (e.g., $V_{\mathrm{b}}=-3 \mathrm{~V}$ or $F=156 \mathrm{kV} / \mathrm{cm}$ ), our results may represent a step towards implementing quantum error correction, which is essential for fault-tolerant quantum computing, through the continuous ultrafast reinitialization of ancillary spin qubits [4,5] within a scalable solid-state quantum device. A further advantage of our hole-spin initialization is that, unlike other works [11-15], it can be easily extended to include initialization of an arbitrary coherent superposition of spin eigenstates by simply controlling the polarization of the laser. Finally, we note that our results should only be taken as a proof-of-principle demonstration that, through further device optimization of the QD, barrier, and bias-voltage switching speed, may open the way for ultrafast high-fidelity initialization of a QD spin qubit without magnetic fields in a future practical quantum computer.

The authors gratefully acknowledge Professor Colin R. Stanley (University of Glasgow) for providing the wafers. J.D.M. would like to thank the Higher Education Funding Council for England (HEFCE), the Cambridge Commonwealth Trust (CCT), and the Natural Sciences and Engineering Research Council (NSERC) of Canada, for financial support.
[1] D. Loss and D. P. DiVincenzo, Phys. Rev. A 57, 120 (1998).

[2] A. Imamoglu, D. D. Awschalom, G. Burkard, D. P. DiVincenzo, D. Loss, M. Sherwin, and A. Small, Phys. Rev. Lett. 83, 4204 (1999).
[3] Semiconductor Spintronics and Quantum Computation, edited by D. D. Awschalom, D. Loss, and N. Samarth (Springer, Berlin, 2002).

[4] D. P. DiVincenzo, Fortschr. Phys. 48, 771 (2000).

[5] J. Preskill, Proc. R. Soc. London, Ser. A 454, 385 (1998). 
[6] D. Brunner, B. D. Gerardot, P. A. Dalgarno, G. Wüst, K. Karrai, N. G. Stoltz, P. M. Petroff, and R. J. Warburton, Science 325, 70 (2009).

[7] K. De Greve, P. L. McMahon, D. Press, T. D. Ladd, D. Bisping, C. Schneider, M. Kamp, L. Worschech, S. Höfling, A. Forchel, and Y. Yamamoto, Nat. Phys. 7, 872 (2011).

[8] A. Greilich, D. R. Yakovlev, A. Shabaev, Al. L. Efros, I. A. Yugova, R. Oulton, V. Stavarache, D. Reuter, A. Wieck, and M. Bayer, Science 313, 341 (2006).

[9] D. Press, K. De Greve, P. L. McMahon, T. D. Ladd, B. Friess, C. Schneider, M. Kamp, S. Höfling, A. Forchel, and Y. Yamamoto, Nat. Photonics 4, 367 (2010).

[10] H. Bluhm, S. Foletti, I. Neder, M. Rudner, D. Mahalu, V. Umansky, and A. Yacoby, Nat. Phys. 7, 109 (2011).

[11] B. D. Gerardot, D. Brunner, P. A. Dalgarno, P. Öhberg, S. Seidl, M. Kroner, K. Karrai, N. G. Stoltz, P. M. Petroff, and R. J. Warburton, Nature (London) 451, 441 (2008).

[12] M. Atatüre, J. Dreiser, A. Badolato, A Högele, K. Karrai, and A. Imamoglu, Science 312, 551 (2006).

[13] X. Xu, Y. Wu, B. Sun, Q. Huang, J. Cheng, D. G. Steel, A. S. Bracker, D. Gammon, C. Emary, and L. J. Sham, Phys. Rev. Lett. 99, 097401 (2007).

[14] D. Press, T. D. Ladd, B. Zhang, and Y. Yamamoto, Nature (London) 456, 218 (2008).

[15] T. M. Godden, S. J. Boyle, A. J. Ramsay, A. M. Fox, and M. S. Skolnick, Appl. Phys. Lett. 97, 061113 (2010).

[16] K. C. Nowack, F. H. L. Koppens, Yu. V. Nazarov, and L. M. K. Vandersypen, Science 318, 1430 (2007).

[17] A. Majumdar, Z. Lin, A. Faraon, and J. Vučković, Phys. Rev. A 82, 022301 (2010).

[18] J. Hansom, C. H. H. Schulte, C. Le Gall, C. Matthiesen, E. Clarke, M. Hugues, J. M. Taylor, and M. Atatüre, Nat. Phys. 10, 725 (2014).

[19] D. V. Bulaev and D. Loss, Phys. Rev. Lett. 95, 076805 (2005).

[20] D. Heiss, S. Schaeck, H. Huebl, M. Bichler, G. Abstreiter, J. J. Finley, D. V. Bulaev, and D. Loss, Phys. Rev. B 76, 241306(R) (2007).

[21] A. J. Ramsay, S. J. Boyle, R. S. Kolodka, J. B. B. Oliveira, J. Skiba-Szymanska, H.Y. Liu, M. Hopkinson, A. M. Fox, and M. S. Skolnick, Phys. Rev. Lett. 100, 197401 (2008).
[22] M. Bayer, G. Ortner, O. Stern, A. Kuther, A. A. Gorbunov, A. Forchel, P. Hawrylak, S. Fafard, K. Hinzer, T. L. Reinecke, S. N. Walck, J. P. Reithmaier, F. Klopf, and F. Schäfer, Phys. Rev. B 65, 195315 (2002).

[23] J. D. Mar, X. L. Xu, J. J. Baumberg, F. S. F. Brossard, A. C. Irvine, C. Stanley, and D. A. Williams, Phys. Rev. B 83, 075306 (2011).

[24] J. D. Mar, X. L. Xu, J. J. Baumberg, A. C. Irvine, C. Stanley, and D. A. Williams, J. Appl. Phys. 110, 053110 (2011).

[25] J. D. Mar, X. L. Xu, J. J. Baumberg, A. C. Irvine, C. Stanley, and D. A. Williams, Appl. Phys. Lett. 99, 031102 (2011).

[26] J. D. Mar, J. J. Baumberg, X. L. Xu, A. C. Irvine, C. R. Stanley, and D. A. Williams, Phys. Rev. B 87, 155315 (2013).

[27] See Supplemental Material at http://link.aps.org/supplemental/ 10.1103/PhysRevB.90.241303 for descriptions of the experimental methods and techniques used for PC measurements of the $X^{0}$ and $X^{+}$spectra, $\Gamma_{e}, \Gamma_{h}$, and $\delta_{\mathrm{FS}}$.

[28] S. M. Sze, Physics of Semiconductor Devices (Wiley, New York, 1981)

[29] M. A. Cusack, P. R. Briddon, and M. Jaros, Phys. Rev. B 56, 4047 (1997).

[30] R. J. Young, R. M. Stevenson, A. J. Shields, P. Atkinson, K. Cooper, D. A. Ritchie, K. M. Groom, A. I. Tartakovskii, and M. S. Skolnick, Phys. Rev. B 72, 113305 (2005).

[31] R. M. Stevenson, R. J. Young, P. See, D. G. Gevaux, K. Cooper, P. Atkinson, I. Farrer, D. A. Ritchie, and A. J. Shields, Phys. Rev. B 73, 033306 (2006).

[32] S. Fafard and C. Nì. Allen, Appl. Phys. Lett. 75, 2374 (1999).

[33] J. D. Mar, X. L. Xu, J. S. Sandhu, A. C. Irvine, M. Hopkinson, and D. A. Williams, Appl. Phys. Lett. 97, 221108 (2010).

[34] K. Müller, A. Bechtold, C. Ruppert, C. Hautmann, J. S Wildmann, T. Kaldewey, M. Bichler, H. J. Krenner, G. Abstreiter, M. Betz, and J. J. Finley, Phys. Rev. B 85, 241306(R) (2012).

[35] W. H. Chang, T. M. Hsu, C. C. Huang, S. L. Hsu, C. Y. Lai, N. T. Yeh, T. E. Nee, and J. I. Chyi, Phys. Rev. B 62, 6959 (2000).

[36] K. H. Schmidt, G. Medeiros-Ribeiro, M. Oestreich, P. M. Petroff, and G. H. Döhler, Phys. Rev. B 54, 11346 (1996).

[37] E. Kadantsev and P. Hawrylak, Phys. Rev. B 81, 045311 (2010). 